\title{
Inverse molecular docking reveals a novel function of thymol: inhibition of fat deposition induced by high-dose glucose in Caenorhabditis elegans
}

\section{Fangfang Ban}

Henan Institute of Science and Technology

Liangbin $\mathrm{Hu}$

Shaanxi University of Science and Technology

Xiaohui Zhou

University of Connecticut

Yanyan Zhao

Henan Institute of Science and Technology

Haizhen Mo

Shaanxi University of Science and Technology

Hongbo Li

Shaanxi University of Science and Technology

Zhou Wei ( $\boldsymbol{D} 15737315032 @ 163 . c o m)$

Henan Institute of Science and Technology

Research

Keywords: inverse molecular docking, thymol, Caenorhabditis elegans, fat

Posted Date: September 21st, 2020

DOl: https://doi.org/10.21203/rs.3.rs-80188/v1

License: (9) This work is licensed under a Creative Commons Attribution 4.0 International License. Read Full License

Version of Record: A version of this preprint was published at Food Science \& Nutrition on June 11th, 2021. See the published version at https://doi.org/10.1002/fsn3.2392. 


\section{Abstract}

Background As a natural product isolated from thyme oil in thyme, thymol (2-isopropyl-5-methylphenol) harbors antiviral, antioxidant, and other properties, and thus could be potentially used for the treatment of various diseases. However, the function of thymol has not been comprehensively studied.

Methods Here, we applied an inverse molecular docking approach to identify unappreciated functions of thymol. Potential targets of thymol in humans were identified by the server of DRAR-CPI, and targets of interest were then assessed by GO and KEGG pathway analysis. Subsequently, homologous proteins of these targets in Caenorhabditis elegans were identified by Blast tool and their three-dimensional structures were achieved using Swiss-Model workspace. Interaction between thymol and the targeted proteins in worms was verified using AutoDock 4.0. To verify the activity of thymol on lipid deposition in vivo, the $C$. elegans model was established. The lipid content of nematodes induced by high-dose glucose was determined by Oil Red $O$ and Nile Red staining, and gene expression related to fat accumulation was assessed by qRT-PCR.

Results Analyses of the targets revealed that thymol could be potentially involved in the glycolysis/gluconeogenesis and fatty acid degradation pathways. Experiment results in vivo verified that $1 \mu \mathrm{g} / \mathrm{mL}$ and $5 \mu \mathrm{g} / \mathrm{mL}$ thymol treatment significantly reduced lipid deposition in glucose-induced nematodes. The result of qRT-PCR suggested that the decreased level of cpt-1, aco, fabp and tph-1 correlating with $\beta$-oxidation in nematodes exposed by $1 \mathrm{mM}$ glucose were upregulated after the exposure of thymol.

Conclusions The results showed that thymol might lead to the acceleration of $\beta$-oxidation by upregulating $c p t-1, a c o$, fabp and tph-1, causing the descent of lipid content in nematodes, which is potentially used for the treatment of chronic metabolic diseases associated with increased fatty acid deposition.

\section{Background}

Metabolic diseases, such as obesity and type-2 diabetes, have been becoming severe health problems. Obesity is considered to be the result of multiple genetic factors and environmental influences. In modern society, foods and drinks rich in high energy including fat and sugar can be seen everywhere. High-fat and high-sugar diet has become the main diet structure at present, and is considered as one of the main causes of obesity caused by the environment.

However, sugar-induced obesity is different from fat-induced obesity. On the one hand, sugar can inhibit fat degradation, on the other hand, it also can be oxidized and decarboxylated to acetyl-CoA that is the materials for the synthesis of fatty acid. As we have known that glycolysis glucose is metabolized to acetyl-CoA from pyruvate by pyruvate dehydrogenase under aerobic conditions, and acetyl-CoA is the substrate of lipid biosynthesis. The rising blood glucose level triggers the release of insulin, leading to the dephosphorylation and activation of acetyl-CoA carboxylase (ACC) by insulin-dependent protein phosphatase. ACC catalyzes the formation of the first intermediate "malonyl-CoA" of fatty acid synthesis, 
which suppresses carnitine acyltransferase I, thereby preventing fatty acid entry into the mitochondrial matrix for degradation through $\beta$-oxidation ${ }^{[1]}$.

Recently more national authorities and the World Health Organization (WHO) have provided advice on the daily diets urging a limited consumption of rapidly digestible starches and sugars ${ }^{[2]}$. Low-energy diet and regular physical exercise are generally considered as a safe way to prevent obesity. Besides, star molecular conjugated linoleic acid (CLA) has been widely applied to adjuvant therapy for chronic metabolic disease. CLA plays a role in the reduction of lipids storage, synthesis, and adipogenesis in adipocytes, and the enhanced fat utilization in muscle via fatty acid $\beta$-oxidation ${ }^{[3]}$. However, some researchers have expressed concerns regarding the safety of CLA for humans ${ }^{[4-7]}$. The main concerns were the involvement of CLA in fatty liver, lipodystrophy, oxidative stress, and glucose intolerance ${ }^{[7-10]}$. Therefore, it is essential to identify alternatives to interfere fat deposition.

Natural essential oil constituents present promising potential to treat infection ${ }^{[11]}$, cancer ${ }^{[12]}$, chronic inflammation ${ }^{[13]}$, and even Alzheimer's disease ${ }^{[14]}$. These constituents cover a group of biologically active molecules, including monoterpenes, sesquiterpenes, oxygenated monoterpenes, oxygenated sesquiterpenes and phenolics among others. As one of the major constituents of essential oil in Monarda punctate, thymol (2-isopropyl-5-methylphenol) has antimicrobial ${ }^{[15]}$, antioxidant ${ }^{[16]}$, antiviral ${ }^{[17]}$, antitumor ${ }^{[18]}$, and anti-inflamatory ${ }^{[13]}$ properties. More importantly, evaluations from the Joint FAO/WHO Expert Committee on Food Additives (JECFA) have indicated that there are no safety concerns on thymol in terms of acceptable daily intake (ADI). Although multiple functions of thymol have been revealed, a comprehensive analysis of thymol's functionality has not been conducted. Computation methodologies for the repositioning of drug molecules onto their receptors have been extensively utilized to reveal novel functions of small chemical compounds. Through reverse screening, we found that thymol may be involved in the regulation of obesity. At present, studies on the effect of thymol on obesity are few and mainly focus on lipid-induced obesity. The effect of thymol on sugar-induced obesity has not been studied. This study will start from this point, and further through the analysis of the glucose metabolism pathway to find the potential target of the action of thymol.

\section{Materials And Methods}

Strains and chemicals. The wild-type $C$. elegans strain N2 was obtained from the $C$. elegans Genetics Center (University of Minnesota, Minneapolis, MN, USA). The eggs of worms were incubated on nematode growth medium (NGM) agar plates at $20^{\circ} \mathrm{C}$, seeded with a live Escherichia coli OP50 (E. coli OP50) as food resources.

Inverse molecular docking of thymol with human proteins. DRAR-CPI server, a tool of inverse molecular docking, was developed to predict the drug target based on structural characteristics of protein ${ }^{[19]}$. According to the algorithm in molecular docking program DOCK, the potential binding sites of receptor molecule in DRAR-CPI are defined by overlapping spheres, consequently, achieving the reverse docking of 
the drug with these binding sites. Affinity for interaction energy in ligand-receptors is evaluated by scoring function and then ranked to predict the potential targets. In this server, potential targets of a ligand can be obtained by submitting a mol2 formatted ligand file. In our study, the mol2-format thymol file was downloaded from the following ZINC online website: (https://zinc.docking.org/substance/1529592).

Functional analysis for target genes. A PPI network was constructed to reveal the hub genes of the potential target proteins on STRING (https://string-db.org/cgi/input.pl), a web portal for undermining the integrated function of multiple genes ${ }^{[20]}$. Besides, on STRING, Gene Ontology (GO) enrichment tool including Biological processes (BPs), cellular components (CCs) and molecular functions (MFs) was used to perform functional analysis of target proteins. Similarly, the Kyoto Encyclopedia of Gene and Genomes (KEGG) enrichment tool was used to analyze biological pathway enrichment to identify those pathways that responded to thymol. It should be noted that the protein name achieved from DRAR-CPI needs to be changed to discernible gene name by UniProtKB before submitting to STRING. Network Modularization analysis was carried by MCODE, a Cytoscape plugin that can be used to identify clusters (highly interconnected regions) in a network.

\section{Blast with $C$. elegans proteins and homologous modeling. Proteins associated with}

Glycolysis/Gluconeogenesis and fatty acid degradation pathway were picked, and their homologs in nematodes were identified by Blast analysis (https://blast.ncbi.nlm.nih.gov/Blast.cgi) ${ }^{[21]}$. Threedimensional structures of these homologs were obtained through homology modeling using SWISSMODEL (https://www.swissmodel.expasy.org/) ${ }^{[22]}$.

Analysis of inverse docking and targeting sites prediction. Inverse docking was analyzed by Autodock 4.0, including AutoGrid and AutoDock ${ }^{[23]}$. AutoGrid calculates related energy in a grid containing Van der Waals force, electrostatic force and hydrogen bond force, while AutoDock conducts conformational search and evaluation using the Lamarckian Genetic Algorithm. For example, the docking of thymol with GAPDH could be realized by the following steps. The first step is the preparation of the system's coordinate files (PDBQT file) of GAPDH and thymol, including the removal of $\mathrm{H}_{2} \mathrm{O}$ (Eliminate the interference of solvation effect) and the addition of hydrogens (X-ray diffraction technology cannot obtain the coordinate data for $\mathrm{H}$ ) in GAPDH molecule, and the setting of active keys number in thymol. The second step is the setting of search sites, mainly including the adjustment of grid size in 'Grad Box'. The third step is the establishment and operation of Autogrid parameter file with GPF format for thymol, generating GLG file and a series of MAP files of GAPDH that contain information of van der Waals forces, electrostatic forces and dissolvent forces of different atom probe et al. The fourth step is the construction and running of Autodock parameter file with DPF format for thymol. Finally, we will achieve a DLG file of thymol. Docking file of GAPDH and thymol should be prepared for Pymol analysis, including the selection of thymol with the lowest energy, the sequence matching for GAPDH and thymol in Notepad++. In the end, a PDB file from Notepad + + needs to be opened in Pymol for the visualization and analysis of GAPDH-thymol docking. We can adjust the appearance of their conformation and search targeting site of thymol by observing its hydrogen bonding site in Pymol. 
Fat staining in $C$. elegans. Worms at characteristic L4 larvae stage were chosen for synchronization. The procedure for glucose induction treatment was carried out as described previously ${ }^{[24]}$. Briefly, $1 \mathrm{mM}$ and $5 \mathrm{mM}$ glucose were added to the $50{ }^{\circ} \mathrm{C}$ molten NGM medium. To determine the effect of thymol on lipid deposition, $1 \mu \mathrm{g} / \mathrm{mL}$ and $5 \mu \mathrm{g} / \mathrm{mL}$ of thymol were added to the NGM medium. The lipid content in the nematodes was evaluated by Oil Red O (ORO) (sigma) staining as described previously [24]. C. elegans at L4s were permeabilized through fixation, freezing and thawing, and dehydration process. Permeabilized worms were stained with ORO in dark overnight at $200 \mathrm{r} / \mathrm{min}, 28^{\circ} \mathrm{C}$. Decolorization of nematodes was then performed with 1, 2- propylene glycol and washed with PBS buffer. The retention of ORO in nematodes could be observed with a $5 \times$ anatomical lens. The quantitative analysis was carried by the method of elution by ethanol. Specifically, quantified nematodes were eluted with absolute ethyl alcohol after discarding PBS buffer by centrifugation and the collected supernatant was transferred to 96-well plate to measure the adsorption of ORO at $510 \mathrm{~nm}$ using a microreader.

Nile Red (Sigma) staining was also performed as described ${ }^{[25]}$. Briefly, $0.5 \mathrm{~mL}$ of the freshly diluted Nile Red $(1 \mu \mathrm{g} / \mathrm{mL})$ was added to plates seeded with E.coli OP50 and allowed to equilibrate for a minimum of $2 \mathrm{~h}$. Then, L1s obtained from bleaching were transferred to the plates and allowed to grow for the desired length of time. Stained nematodes were imaged under a $10 \times$ objective under dark field and red fluorescence illumination with an inverted fluorescent microscope.

RNA isolation and qRT-PCR. The total RNA of nematodes was isolated using TRIzol $\circledast$ Reagent (Invitrogen) and purified with DNase I, RNase-free (Thermo Scientific ${ }^{T M}$ ). cDNA was obtained using MMuLV First Strand cDNA Synthesis Kit (Sangon, Shanghai, China). Real-time PCR was performed using SYBR Green $₫$ probes by QuantStudio ${ }^{\circledR} 3$ System ${ }^{[26]}$. Primer sequences were listed in Table S1. Relative expression of genes was determined using the $2-\Delta \Delta C$ t method and act- 1 was chosen as the reference gene.

Statistics analysis. All assays were repeated independently at least 3 times, and the experimental data were represented as mean \pm SD. The data between two different treatments were compared statistically by one-way ANOVA in SPSS. The significance of differences was defined at the $P<0.05$ level and $P<$ 0.01 .

\section{Results}

Protein targets prediction and analysis. Analysis by DRAR-CPI servers revealed that 392 human proteins have potential interaction with thymol. In KEGG pathway, we found interesting pathways including glycolysis/gluconeogenesis and fatty acid degradation pathway relative to obesity. Further analysis indicated that targets in these pathways associated with carbon metabolism, tyrosine metabolism, pyruvate metabolism, retinol metabolism, drug metabolism-cytochrome p450, metabolism of xenobiotics by cytochrome p450, biosynthesis of amino acids and chemical carcinogenesis pathways (Fig. 1A). In addition, these targets mainly localized in the cytosol (Fig. 1B) and associated with coenzyme binding, oxidoreductase activity, catalytic activity, oxidoreductase activity, acting on the $\mathrm{CH}-\mathrm{OH}$ group of donors, 
NAD or NADP as acceptor and alcohol dehydrogenase activity, zinc-dependent (Fig. 1C), and involved in organic substance catabolic process, single-organism biosynthetic process, small molecule metabolic process, xenobiotic process, ethanol metabolic process, pyruvate metabolic process, glucose metabolic process, oxidation-reduction process and ethanol process (Fig. 1D).

Our analysis also showed that module 1 (Fig. 1E) was the most important in the whole PPI network, with the highest score of 19.714. GAPDH (Glyceraldehyde-3-phosphate dehydrogenase) is a node in module 1 with the MCODE - score of 12.72, which has the highest complexity in 12 proteins responding to thymol, predicting it may be the central protein in glycolysis. Independent of its glycolytic activity, the encoded protein has additionally been identified to have uracil DNA glycosylase activity in the nucleus ${ }^{[27]}$. Also, GAPDH harbors a region that confers antimicrobial activity against Staphylococcus bacteria ${ }^{[28]}$. Studies of a similar protein in mice have assigned a variety of additional functions including nitrosylation of nuclear proteins, the regulation of mRNA stability, and acting as a transferrin receptor on the cell surface of macrophage ${ }^{[29-31]}$. Module 2 (Fig. 1F) had a score of 4.939 and included ADH1A and ADH1B nodes with the MCODE-score of 11 respectively. Module 3 (Fig. 1G) had a score of 3.636 and was involved in the TPI note. Module 4 had a score of 3.273 and included three nodes involved in it including PKLR, LDHB, and ADH7 (Fig. 1H). Besides, two other proteins (ACAT1 and FBP1) were not present in the module. The functional annotation of these representative proteins and reported experimental correlation with thymol were described as Table 1. 
Table 1

Function annotation of potential targets of thymol

\begin{tabular}{|c|c|c|c|c|}
\hline $\begin{array}{l}\text { PDB } \\
\text { ID }\end{array}$ & Protein Name & $\begin{array}{l}\text { Predicted } \\
\text { Docking } \\
\text { Score }\end{array}$ & $\begin{array}{l}\text { Protein Function and Reported Connection } \\
\text { with Diseases }\end{array}$ & $\begin{array}{l}\text { Reported } \\
\text { Experimental } \\
\text { Correlation } \\
\text { with thymol }\end{array}$ \\
\hline 1ZNQ & $\begin{array}{l}\text { Glyceraldehyde- } \\
\text { 3-phosphate } \\
\text { dehydrogenase }\end{array}$ & -22.3993 & $\begin{array}{l}\text { catalyzes the reversible oxidative } \\
\text { phosphorylation in the presence of } \\
\text { inorganic phosphate and nicotinamide } \\
\text { adenine dinucleotide (NAD) in } \\
\text { carbohydrate metabolism. Suppresses } \\
\text { proliferation and invasion of lung and } \\
\text { esophageal squamous cell carcinomas } \\
\text { [40]. }\end{array}$ & No \\
\hline 1HSO & $\begin{array}{l}\text { Alcohol } \\
\text { dehydrogenase } \\
1 \mathrm{~A}\end{array}$ & -23.3497 & $\begin{array}{l}\text { catalyzes the oxidation of alcohols to } \\
\text { aldehydes. Variant confers susceptibility } \\
\text { to esophageal squamous-cell carcinoma } \\
\text { [41]. } \\
\text { Polymorphisms in ADH1B associated with } \\
\text { the increased risk of gastric cancer }{ }^{[42]} \text {. }\end{array}$ & No \\
\hline $1 \mathrm{HSZ}$ & $\begin{array}{l}\text { Alcohol } \\
\text { dehydrogenase } \\
\text { 1B }\end{array}$ & -22.79 & Same as ADH1A & No \\
\hline 1D1T & $\begin{array}{l}\text { Alcohol } \\
\text { dehydrogenase } \\
\text { class } 4 \\
\text { mu/sigma chain }\end{array}$ & -25.5514 & $\begin{array}{l}\text { Same as ADH1A and ADH1B, however, the } \\
\text { enzyme is inefficient in ethanol oxidation, } \\
\text { but it is the most active as a retinol } \\
\text { dehydrogenase, thus it may participate in } \\
\text { the synthesis of retinoic acid, a hormone } \\
\text { important for cellular differentiation. A } \\
\text { single nucleotide polymorphism ADH7 is } \\
\text { associated with the risk of squamous cell } \\
\text { carcinoma of the head and neck }\end{array}$ & No \\
\hline $110 Z$ & $\begin{array}{l}\text { L-lactate } \\
\text { dehydrogenase B } \\
\text { chain }\end{array}$ & -25.1132 & $\begin{array}{l}\text { catalyzes the interconversion of pyruvate } \\
\text { and lactate with concomitant } \\
\text { interconversion of NADH and NAD }{ }^{+} \text {in a } \\
\text { post-glycolysis process. Correlates With } \\
\text { unfavorable survival in hepatocellular } \\
\text { carcinoma }{ }^{[44]} \text {, and promotion of } \\
\text { pancreatic cancer progression }{ }^{[45]} \text {. }\end{array}$ & No \\
\hline 2VGB & $\begin{array}{l}\text { Pyruvate kinase } \\
\text { PKLR }\end{array}$ & -24.1893 & $\begin{array}{l}\text { Catalyzes the transphosphorylation of } \\
\text { phohsphoenolpyruvate into pyruvate and } \\
\text { ATP, which is the rate-limiting step of } \\
\text { glycolysis. } \\
\text { Correlates the promotion colorectal cancer } \\
\text { liver colonization }{ }^{[46]} \text {. }\end{array}$ & No \\
\hline
\end{tabular}




\begin{tabular}{|lllll|}
\hline $\begin{array}{l}\text { PDB } \\
\text { ID }\end{array}$ & Protein Name & $\begin{array}{l}\text { Predicted } \\
\text { Docking } \\
\text { Score }\end{array}$ & $\begin{array}{l}\text { Protein Function and Reported Connection } \\
\text { with Diseases }\end{array}$ & $\begin{array}{l}\text { Reported } \\
\text { Experimental } \\
\text { Correlation } \\
\text { with thymol }\end{array}$ \\
\hline $1 \mathrm{HTI}$ & $\begin{array}{l}\text { Triosephosphate } \\
\text { isomerase }\end{array}$ & -22.7411 & $\begin{array}{l}\text { Catalyzes the isomerization of } \\
\text { glyceraldehydes 3-phosphate (G3P) and } \\
\text { dihydroxy-acetone phosphate (DHAP) in } \\
\text { glycolysis and gluconeogenesis. Variant }\end{array}$ & No \\
\hline (Arg189GIn) causes neurologic deficits \\
[47].
\end{tabular}

Molecular docking. To confirm the activity of thymol on lipid synthesis and metabolism in vivo, we chosen nematodes as experimental models due to their advantages such as a short cycle, a fast reproduction rate, and high homology with human genes. Homologous genes of thymol's targets in nematodes were identified by Blast analysis (https://blast.ncbi.nlm.nih.gov/Blast.cgi). These analyses revealed GPD-1, GPD-2, GPD-3, GPD-4, H24K24.3, TPI-1, PYK-1, PYK-2, 1DH-1, KAT-1, T02G5.7 and FBP-1 in nematodes. However, their 3D structures remain unknown except for TPI-1. Therefore, we constructed the $3 \mathrm{D}$ structure of the remaining protein by homology modeling technology. Homology modeling is a technique that can build a 3D structure for proteins based on the 3D structure of a similar protein. A homology model can provide an alternative for subsequent receptor-ligand analyses such as molecular docking.

In our study, we constructed models of these homology proteins by SWISS-MOL and examined interactions between thymol and these proteins through Autodock 4.0 (Fig. 2). Docking analysis revealed that thymol potentially interacts with the different amino acids of these proteins (except 1DH-1) in nematodes (Table 2). 
Table 2

Potential targets and corresponding amino acid action site of thymol

\begin{tabular}{|c|c|c|}
\hline Potential targets & $\begin{array}{c}\text { Corresponding amino acid } \\
\text { action site }\end{array}$ & $\begin{array}{l}\text { Three-dimensional } \\
\text { structure interaction } \\
\text { diagrams }\end{array}$ \\
\hline GPD-1 & Gly 209 & \\
\hline GPD-2 & Lys 57 and Arg 20 & \\
\hline GPD-3 & Pro 242 & \\
\hline GPD-4 & $\mathrm{G} \ln 210$ & \\
\hline $\mathrm{H} 24 \mathrm{~K} 24.3$ & Thr 326 and Thr 191 & \\
\hline PYK-2 & Asn 17 and Lys 377 & \\
\hline $1 \mathrm{DH}-1$ & Asn 273 & \\
\hline KAT-1 & Ala 290 and Val 27 & \\
\hline T02G5.7 & Ser 248 & \\
\hline $1 \mathrm{MO} 0$ & Arg 204 and Ile 159 & \\
\hline
\end{tabular}

Thymol reduces fat deposition due to high glucose exposure in nematodes. Fat accumulation induced by high-dose glucose ( $1 \mathrm{mM}$ or $5 \mathrm{mM}$ ) in nematodes is inheritable across several generations ${ }^{\text {[24] }}$. So we chose $1 \mathrm{mM}$ or $5 \mathrm{mM}$ of glucose to induce fat deposition in nematodes. ORO allows for the quantification of total lipid levels, while Nile red facilitates the evaluation of lipid distribution among tissues such as the hypodermis, intestine, and the germ line. When used in conjunction, ORO and Nile Red enable the researchers to determine how genotype and environmental changes affect lipid accumulation and where in the worm these changes are occurring ${ }^{[32]}$. The statistic result of ORO and Nile Red staining suggests both $1 \mathrm{mM}$ and $5 \mathrm{mM}$ of glucose are capable of inducing fat accumulation in the second generation of 
nematodes, and $5 \mathrm{mM}$-induced fat accumulation is more significant than $1 \mathrm{mM}$-induced in nematode (Fig. 3B). Total lipid levels and lipid droplets were visualized by staining with ORO (Fig. 3A) and Nile Red (Fig. 3C) respectively. Futher, the treatment of thymol can reduce lipid content. Importantly, $1 \mu \mathrm{g} / \mathrm{mL}$ and $5 \mu \mathrm{g} / \mathrm{mL}$ thymol treatment significantly reduced lipid deposition in glucose-induced nematodes, indicating thymol can reduce fat content.

Thymol restores the change of transcriptional level of genes in $\beta$-oxidation induced by high-dose glucose. Our experimental data verified thymol could reduce lipid deposition in worms. We mentioned above that glucose-induced fat accumulation is on the one hand due to the synthesis of acetyl-CoA from glucose through glycolysis, which is the substrate of lipid biosynthesis, on the other hand is due to the inhibition of fat degradation by the activation of acetyl-CoA carboxylase (ACC) triggered by the rising blood glucose level. Therefore, we suspect that the reduction of fat accumulation by thymol may be achieved by regulating the upstream of glycolysis pathway and downstream of fatty acid $\beta$ oxidation. To confirm this idea, we determined the transcriptional level of key genes correlating with $\beta$-oxidation including carnitine palmitoyltransferase (cpt-1), acyl-CoA oxidase (aco), fatty acid binding protein $(f a b p)$ in the process of $\beta$ oxidation and tryptophan hydroxylase (tph-1) in the upstream pathway of $\beta$-oxidation. The result of qRTPCR suggested that the decreased level of $c p t-1, a c o, f a b p$ and $t p h-1$ in nematodes exposed by $1 \mathrm{mM}$ glucose were upregulated after the exposure of thymol (Fig. 4). These findings suggest the reduction of fat deposition caused by thymol is related to the oxidation of fatty acids.

\section{Discussion}

In silico approaches can improve our ability to predict drug targets, thereby streamlining and accelerating the discovery process of a novel function of the drug. In this study, we found that thymol has a potential interaction with proteins associated with glycolysis/gluconeogenesis and fatty acid degradation pathway through reverse molecular docking approaches. These proteins included GAPDH, ADH1A, ADH1B, TPI-1, PKLR, ADH7, LDHB, ACAT1, and FBP1 in humans. The homologous proteins in nematode are GPD-1, GPD-2, GPD-3, GPD-4, H24K24.3, TPI-1, PYK-1, PYK-2, 1DH-1, KAT-1, T02G5.7 and FBP-1, respectively. Some of the putative thymol targets have been identified for an association with fat storage previously. For example, kat-1, the major nematodes fat storage tissue, acts in the intestine. The gene transcription

level of kat-1 was up-regulated during fat feeding ${ }^{[33]}$ and down-regulated after hesperidin treatment ${ }^{[26]}$. In nematodes, four genes designated gpd-1 through gpd-4 encode glyceraldehyde-3-phosphate dehydrogenase, and $\triangle g p d-1$ can cause greatly raised triglycerides ${ }^{[34]}$. However, there is no known link between the remaining genes (including PYK-2, TPI-1, 1DH1, T02G5.7, D2063.1, H24K24.3 and SODH-2) and obesity. These provide us with proof that this method has reliability for the prediction of thymol.

Our experimental results in vivo verified that thymol inhibited lipid deposition induced by high-dose glucose in nematodes. To understand how thymol makes a function in inhibiting fat accumulation, we detected the expression level of key genes correlation with $\beta$-oxidation by qRT-PCR. The findings suggested that thymol could recover the changes in the expression levels of these genes induced by highdose glucose, including $c p t-1$, aco, fabp and $t p h-1$. So thymol probably accelerates $\beta$-oxidation according 
to the determination of qRT-PCR combined with bioinformatics prediction. Furthermore, we suppose that thymol may accerlate glucose metabolism by regulating the targets of GPD-1, GPD-2, GPD-3, GPD-4, PYK$2,1 \mathrm{DH}-1,1 \mathrm{MOO}$ in the glycolytic pathway, causing the reduction of blood glucose level and ACC

inactivation. The consequence of ACC inhibition is a reduction in malonyl-CoA, which releases carnitine palmitoyltransferase (CPT) from inhibition and causes the transport of fatty acids into the mitochondria for $\beta$-oxidation. And the protein targets we predicted including H24K24.3, KAT-1 and T02G5.7 in the fatty acid degradation pathway may be located the downstream of key genes correlationg with $\beta$-oxidation (Fig. 5).

\section{Conclusion}

We found interesting pathways including glycolysis pathway and fatty acid degradation pathway correlation with obesity by bioinformatics from database of human beings. Futher analysis indicated that there are the same pathways in C.elegans, and the targets of the pathways including GPD-1, GPD-2, GPD3, GPD-4, PYK-2, 1DH-1, 1MO0, H24K24.3, KAT-1 and T02G5.7 have a pretty interaction with thymol. Experiment results in vivo showed that $1 \mu \mathrm{g} / \mathrm{mL}$ and $5 \mu \mathrm{g} / \mathrm{mL}$ thymol treatment significantly reduced lipid deposition in glucose-induced nematodes, which is consistent with prediction by bioinformation. The result of qRT-PCR suggested that the decreased level of $c p t-1, a c o$, fabp and $t p h-1$ correlating with $\beta$ oxidation in nematodes exposed by $1 \mathrm{mM}$ glucose were upregulated after the exposure of thymol. Together, thymol can reduce lipid acuumulation induced by high-glucose through raising $\beta$-oxidation level of fatty acid.

\section{Abbreviations}

CLA: conjugated linoleic acid

ORO: Oil Red O

cpt-1: carnitine palmitoyltransferase

aco: acyl-CoA oxidase

fabp: fatty acid binding protein

tph-1: tryptophan hydroxylase

ACC: acetyl-CoA carboxylase

\section{Declarations}


Availability of data and materials

All data generated or analyzed during this study are included in this published paper or are available from the corresponding author on reasonable request.

\section{Acknowledgements}

The authors gratefully acknowledge Zhen-Kun Cui for critical review of this manuscript.

\section{Funding}

This work is financially supported by the General Project of National Natural Science Foundation of China (Grant No.31671952), Program for Science \& Technology Innovation Talents in Universities of Henan Province (18HASTIT037).

\section{Author information}

\section{Affiliations}

Department of Food Science, Henan Institute of Science and Technology, Xinxiang, 453003, Henan, China Fang-Fang Ban, Yan-Yan Zhao, Wei Zhou

Department of Food and Bioengineering, Shaanxi University of Science \& Technology, Xi'an, 710021, Shaanxi, China

Liang-Bin Hu, Hai-Zhen Mo, Hong-Bo Li

Department of Pathobiology \& Veterinary Science, University of Connecticut, Mansfield, 06269, Connecticut, United States

Xiao-Hui Zhou

\section{Contributions}

FFB, LBH, HZM, XHZ \& WZ: conceived and designed the experiments as well as wrote the paper; FFB: performed experiments; YYZ \& HBL: Collected and analyzed the data. All authors have read and approved the final manuscript.

\section{Corresponding authors}

Correspondence to Xiao-Hui Zhou or Wei Zhou.

\section{Ethics declarations}


Caenorhabditis elegans do not require committee approvals for experimentation.

\section{Consent for publication}

Not applicable.

\section{Competing interests}

The authors declare that they have no competing interests.

\section{Additional information}

\section{Publisher's Note}

Springer Nature remains neutral with regard to jurisdictional claims in published maps and institutional affiliations.

\section{References}

1. Zheng J, Greenway FL. Caenorhabditis elegans as a model for obesity research. Int J Obes (Lond). 2012; 36: 186-94.

2. Brouns F. Overweight and diabetes prevention: is a low-carbohydrate-high-fat diet recommendable? Eur J Nutr. 2018; 57: 1301-12.

3. Park Y, Pariza MW. Mechanisms of body fat modulation by conjugated linoleic acid (CLA). Food Res Int. 2007; 40: 311-23.

4. Kelley DS, Erickson KL. Modulation of body composition and immune cell functions by conjugated linoleic acid in humans and animal models: benefits vs. risks. Lipids. 2003; 38: 377-86.

5. Larsen TM, Toubro S, Astrup A. Efficacy and safety of dietary supplements containing CLA for the treatment of obesity: evidence from animal and human studies. J Lipid Res. 2003; 44: 2234-41.

6. Riserus U, Basu S, Jovinge S, et al. Supplementation with conjugated linoleic acid causes isomerdependent oxidative stress and elevated C-reactive protein: a potential link to fatty acid-induced insulin resistance. Circulation. 2002; 106: 1925-29.

7. Tricon S, Yaqoob P. Conjugated linoleic acid and human health: a critical evaluation of the evidence. Curr Opin Clin Nutr Metab Care. 2006; 9: 105-10.

8. Clement L, Poirier $\mathrm{H}$, Niot I, et al. Dietary trans-10,cis-12 conjugated linoleic acid induces hyperinsulinemia and fatty liver in the mouse. J Lipid Res. 2002; 43: 1400-9.

9. Pariza MW. Perspective on the safety and effectiveness of conjugated linoleic acid. Am J Clin Nutr. 2004; 79: 1132s-6s.

10. Poirier $\mathrm{H}$, Niot I, Clement L, et al. Development of conjugated linoleic acid (CLA)-mediated lipoatrophic syndrome in the mouse. Biochimie. 2005; 87: 73-79. 
11. Zhang Y, Liu Y, Qiu J, et al. The herbal compound thymol protects mice from lethal infection by Salmonella Typhimurium. Front Microbiol. 2018; 9: 1022.

12. Xie B, Nagalingam A, Kuppusamy $P$, et al. Benzyl isothiocyanate potentiates $p 53$ signaling and antitumor effects against breast cancer through activation of p53-LKB1 and p73-LKB1 axes. Sci Rep. 2017; 7: 40070.

13. Yao L, Hou G, Wang L, et al. Protective effects of thymol on LPS-induced acute lung injury in mice. Microb Pathog. 2018; 116: 8-12.

14. Postu PA, Sadiki FZ, El Idrissi M, et al. Pinus halepensis essential oil attenuates the toxic Alzheimer's amyloid beta (1-42)-induced memory impairment and oxidative stress in the rat hippocampus. Biomed Pharmacother. 2019; 112: 108673.

15. Marchese A, Orhan IE, Daglia M, et al. Antibacterial and antifungal activities of thymol: A brief review of the literature. Food Chem. 2016; 210; 402-14.

16. Luna A, Lema-Alba RC, Dambolena JS, et al. Thymol as natural antioxidant additive for poultry feed: oxidative stability improvement. Poult Sci. 2017; 96: 3214-20.

17. Lai WL, Chuang HS, Lee MH, et al. Inhibition of herpes simplex virus type 1 by thymol-related monoterpenoids. Planta Med. 2012; 78: 1636-8.

18. Gunes-Bayir A, Kocyigit A, Guler EM, et al. Effects of thymol, a natural phenolic compound, on human gastric adenocarcinoma cells in vitro. Altern Ther Health Med. 2019; 25: 12-21.

19. Luo $H$, Chen J, Shi L, et al. DRAR-CPI: a server for identifying drug repositioning potential and adverse drug reactions via the chemical-protein interactome. Nucleic Acids Res. 2011; 39: 492-8.

20. Szklarczyk D, Franceschini A, Wyder S, et al. STRING v10: protein-protein interaction networks, integrated over the tree of life. Nucleic Acids Res. 2015; 43: D447-52.

21. Hu LB, Ban FF, Li HB, et al. Thymol induces conidial apoptosis in Aspergillus flavus via stimulating $\mathrm{K}(+)$ eruption. J Agric Food Chem. 2018; 66: 8530-6.

22. Zong H. Effects of diet - induced lipodeposition on egg laying capacity of nematodes. Hefei university of technology. 2017.

23. Biasini M, Bienert S, Waterhouse A, et al. SWISS-MODEL: modelling protein tertiary and quaternary structure using evolutionary information. Nucleic Acids Res. 2014; 42: W252-w258.

24. Morris GM, Huey R, Lindstrom W, et al. AutoDock4 and AutoDockTools4: Automated docking with selective receptor flexibility. J Comput Chem. 2009; 30: 2785-91.

25. Zhao LJ. Epigenetic study of fat deposition in nematodes induced by glucose. Hefei university of technology. 2015.

26. Fouad AD, Pu SH, Teng S, et al. Quantitative assessment of fat levels in Caenorhabditis elegans using dark field microscopy. G3 (Bethesda, Md.). 2017; 7: 1811-8.

27. Peng H, Wei Z, Luo H, et al. Inhibition of fat accumulation by hesperidin in Caenorhabditis elegans. $J$ Agric Food Chem. 2016; 64: 5207-14. 
28. Meyer-Siegler K, Mauro DJ, Seal G, et al. A human nuclear uracil DNA glycosylase is the 37-kDa subunit of glyceraldehyde-3-phosphate dehydrogenase. Proc Natl Acad Sci USA. 1991;88: 8460-4.

29. Ong JS, Taylor TD, Wong CB, et al. Extracellular transglycosylase and glyceraldehyde-3-phosphate dehydrogenase attributed to the anti-staphylococcal activity of Lactobacillus plantarum USM861. J Biotechnol. 2019; 300: 20-31.29. Kornberg MD, Sen N, Hara MR, Juluri KR, Nguyen JV, Snowman AM, Law L, Hester LD, Snyder SH. GAPDH mediates nitrosylation of nuclear proteins. Nat Cell Biol. 2010; 12: $1094-100$.

30. Rodriguez-Pascual F, inert $H$, Lamas S. Glyceraldehyde-3-phosphate dehydrogenase regulates endothelin-1 expression by a novel, redox-sensitive mechanism involving mRNA stability. Mol Cell Biol. 2008; 28: 7139-55.

31. Raje $\mathrm{Cl}$, Kumar $\mathrm{S}$, Harle A, et al. The macrophage cell surface glyceraldehyde-3-phosphate dehydrogenase is a novel transferrin receptor. J Biol Chem. 2007; 282: 3252-61.

32. Wilber E, Dana LR, James N, et al. Quantitative assessment of fat levels in Caenorhabditis elegans using dark field microscopy. G3 (Bethesda, Md.). 2017; 7: 1811-8.

33. Mak HY, Nelson LS, Basson M, et al. Polygenic control of Caenorhabditis elegans fat storage. Nat Genet. 2006; 38: 363-8.

34. Hegele RA, Ginsberg HN, Chapman MJ, et al. The polygenic nature of hypertriglyceridaemia: implications for definition, diagnosis, and management. Lancet Diabetes Endocrinol. 2014; 2: 65566.

\section{Figures}


A

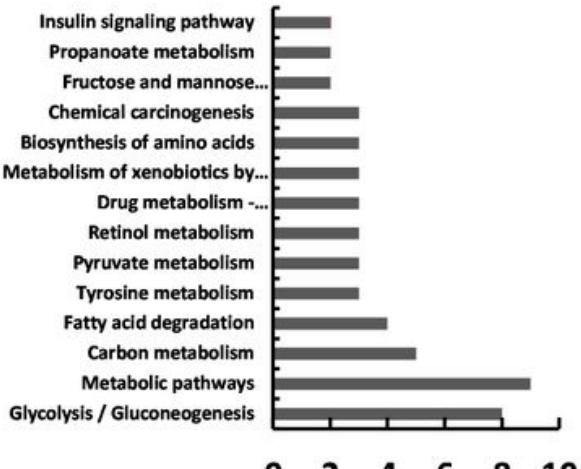

$\begin{array}{llllll}0 & 2 & 4 & 6 & 8 & 10\end{array}$

= observed gene count $=$ false discovery rate

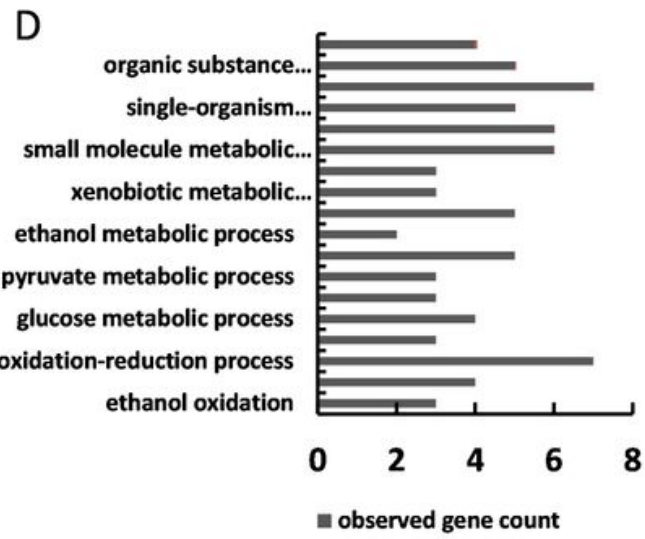

$\mathrm{F}$

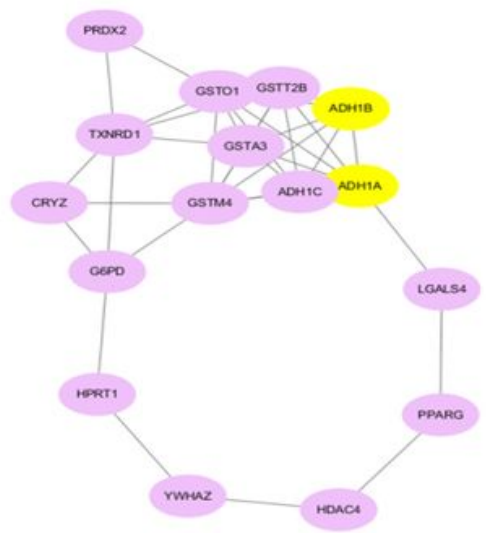

B

C

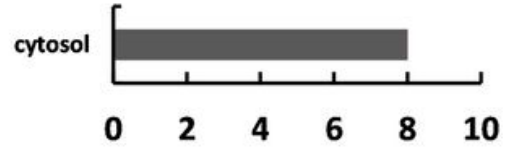

abserved gene count $=$ false discovery rate

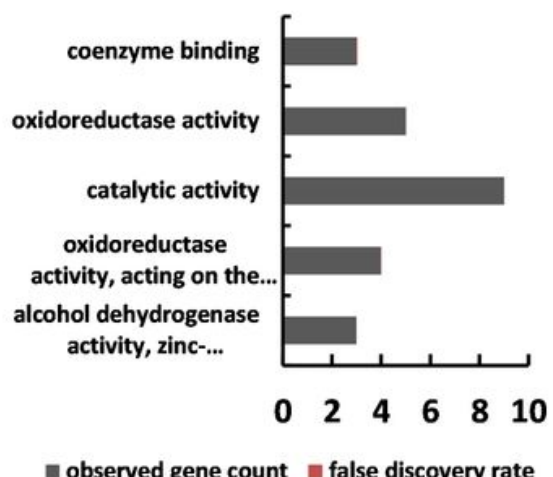

- observed gene count = false discovery rate

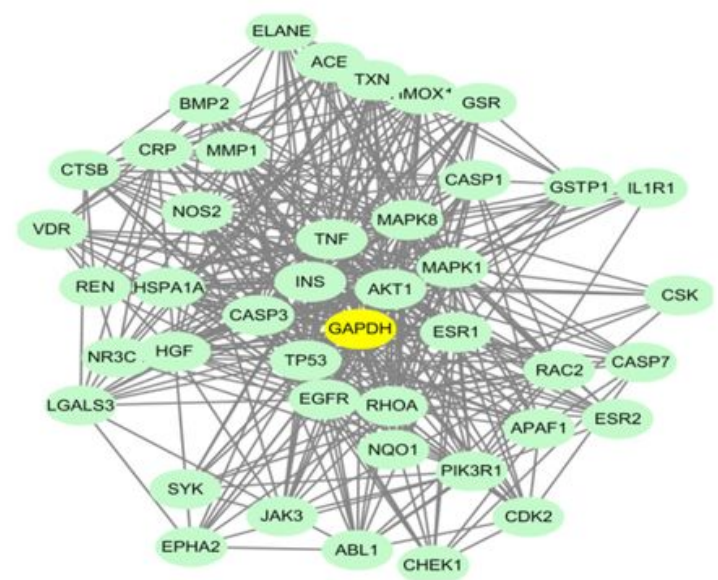

$\mathrm{H}$
G

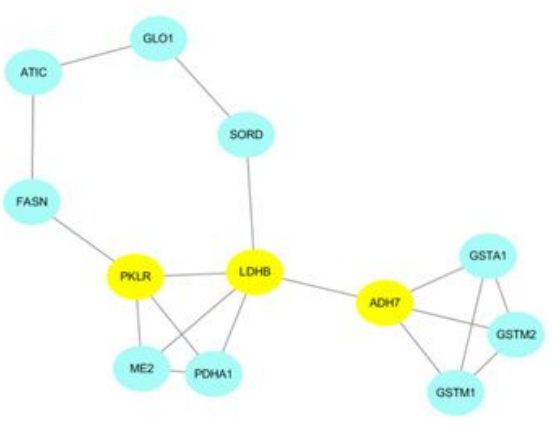

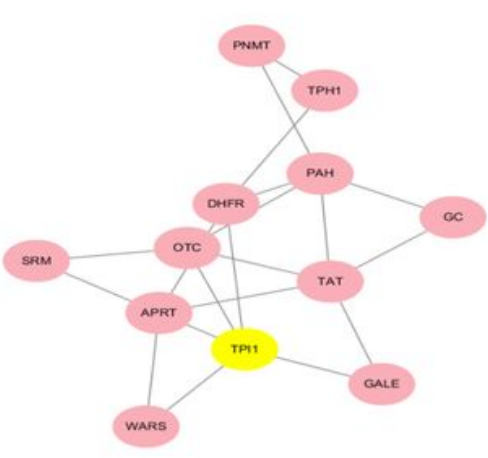

Figure 1

GO and KEGG pathway analysis of potential targets inferred to fat synthesis and metabolism (A) KEGG pathway (B) Cellular components pathway (C) Molecular function pathway (D) biological process pathway. (E) $(F)(G)(H)$ MCODE of proteins of interest in the whole PPI network. 
GPD-1

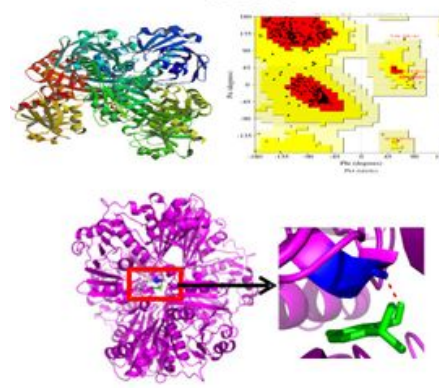

Thymol-GLY 209
GPD-2

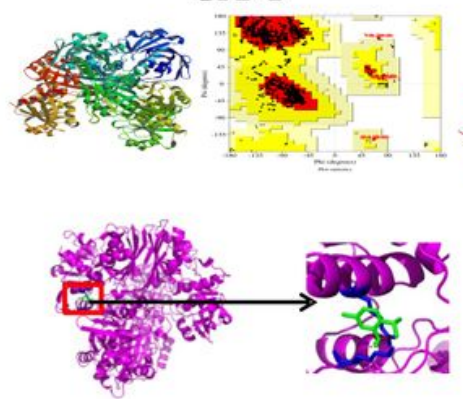

Thymol-GLS 57
GPD-3

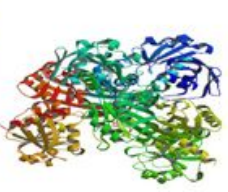

GPD-4
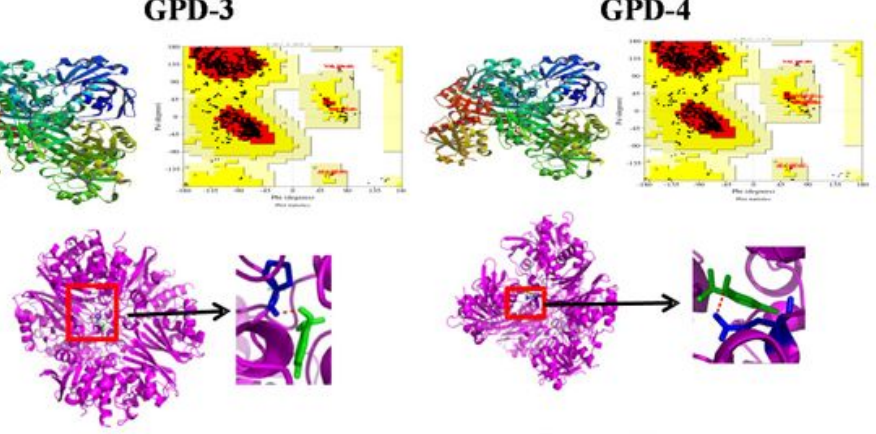

Thymol-PRO 242

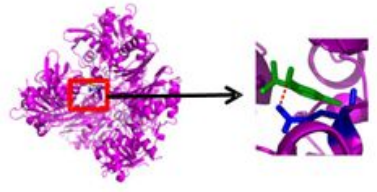

Thymol-GLA 210
H24K24.3

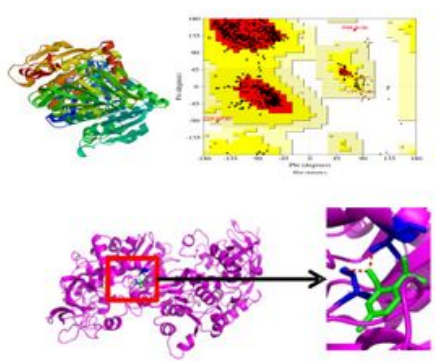

Thymol-THR 326 and THR 191

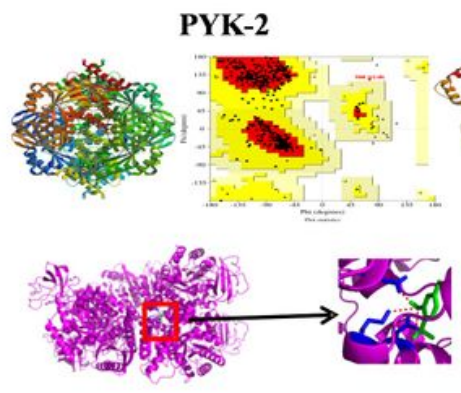

Thymol-ASN 17and LYS 377
1DH-1
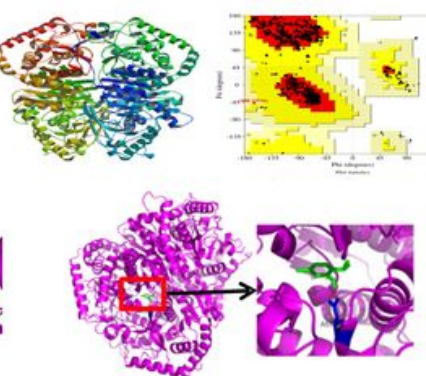

Thymol-ASN 273

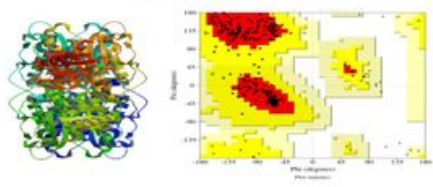

PYK-1

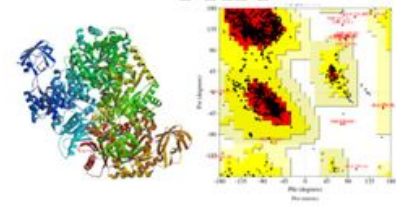

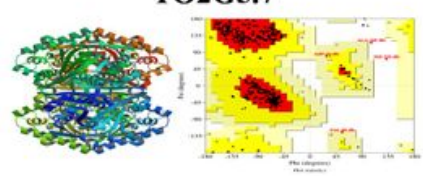

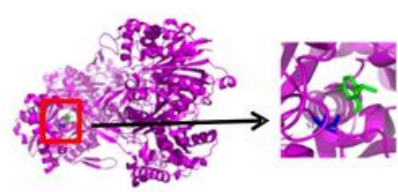

Thymol- SER 248
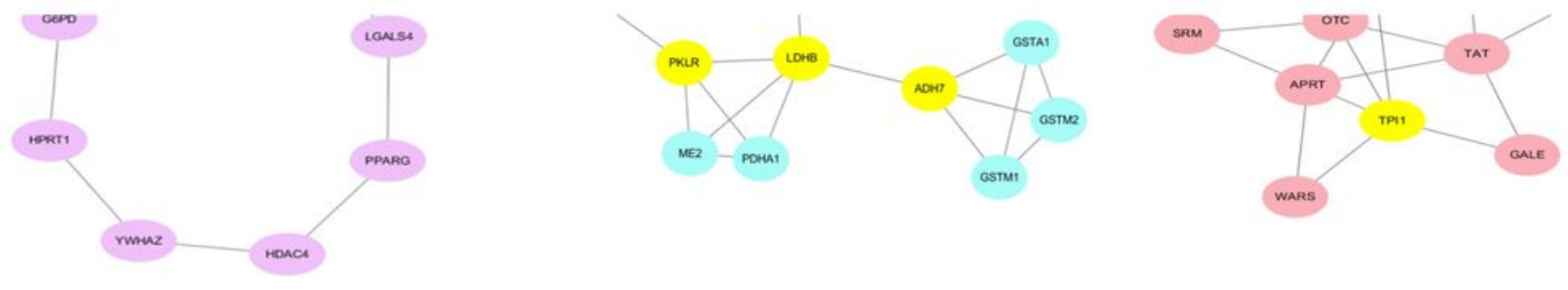

Figure 2

Homologous modeling of homologous proteins in nematodes at SWISS-MODEL network, evaluation of their 3D structure by SAVES and molecular docking between target proteins and thymol through AutoDock 4.0. 
A

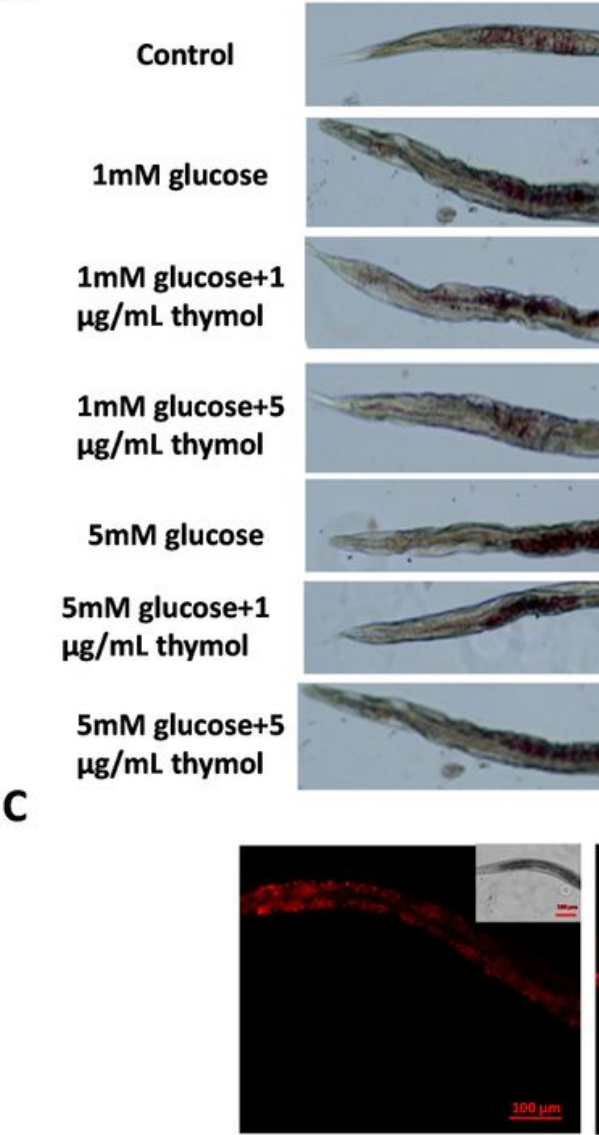

Control

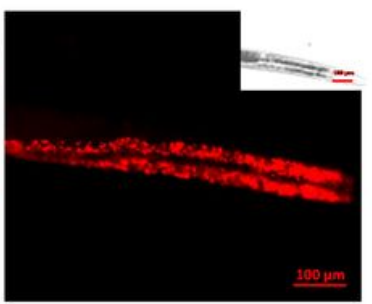

$1 \mathrm{mM}$ glucose
B

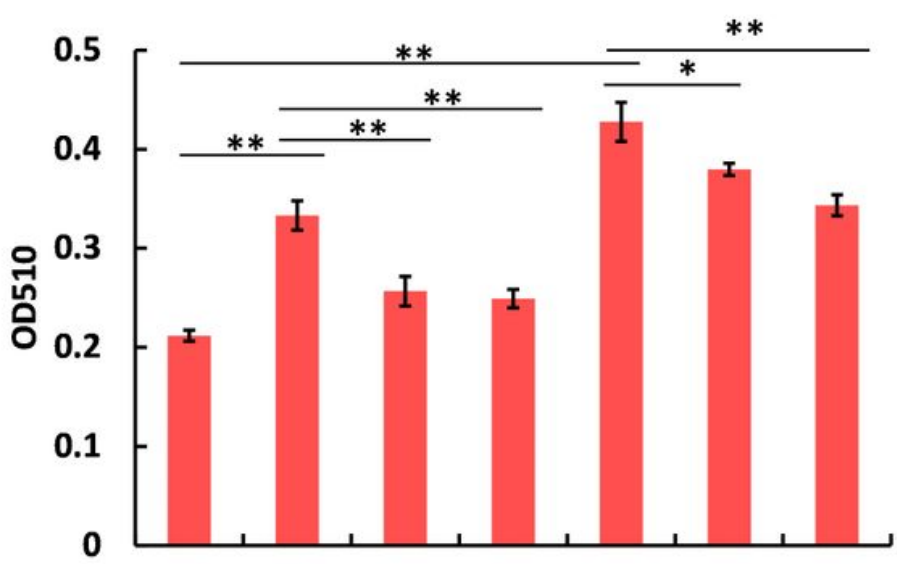

$\begin{array}{llllllll}1 \mathrm{mM} \text { glucose } & - & + & + & + & - & - & - \\ 5 \mathrm{mM} \text { glucose } & - & - & - & - & + & + & + \\ 1 \mu \mathrm{mL} \text { thymol } & - & - & + & + & - & - & - \\ 5 \mu \mathrm{g} / \mathrm{mL} \text { thymol } & - & - & - & - & - & + & +\end{array}$
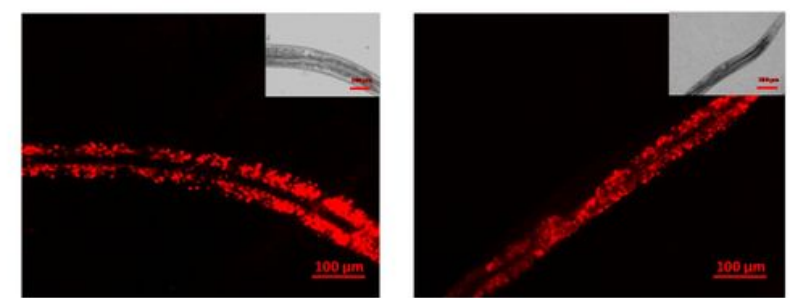

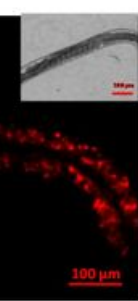

$1 \mathrm{mM}$ glucose + $1 \mu \mathrm{g} / \mathrm{mL}$ thymol

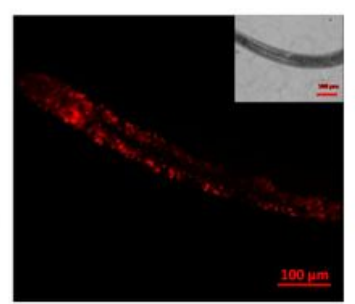

$1 \mathrm{mM}$ glucose + $5 \mu \mathrm{g} / \mathrm{mL}$ thymol
$5 \mathrm{mM}$ glucose +

$1 \mu \mathrm{g} / \mathrm{mL}$ thymol

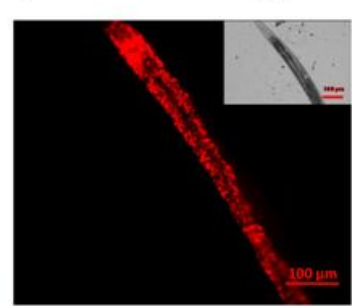

$5 \mathrm{mM}$ glucose +

$5 \mu \mathrm{g} / \mathrm{mL}$ thymol

\section{Figure 3}

The effect of thymol on fat accumulation in nematodes induced by $1 \mathrm{mM}$ and $5 \mathrm{mM}$ glucose respectively. (A) Fat deposition images of glucose-induced nematodes post different concentrations of thymol treatment and stained with Oil Red O. (B) Quantitative analysis of lipid accumulation in glucose-induced nematodes treated with different concentrations of thymol and stained with Oil Red $O$ through alcohol decolorization, ${ }^{\star} P<0.05,{ }^{*} P<0.01$. (C) Fluorescent pictures of glucose-induced nematodes treated with different concentrations of thymol and stained with Nile Red under an inverted fluorescent microscope. 


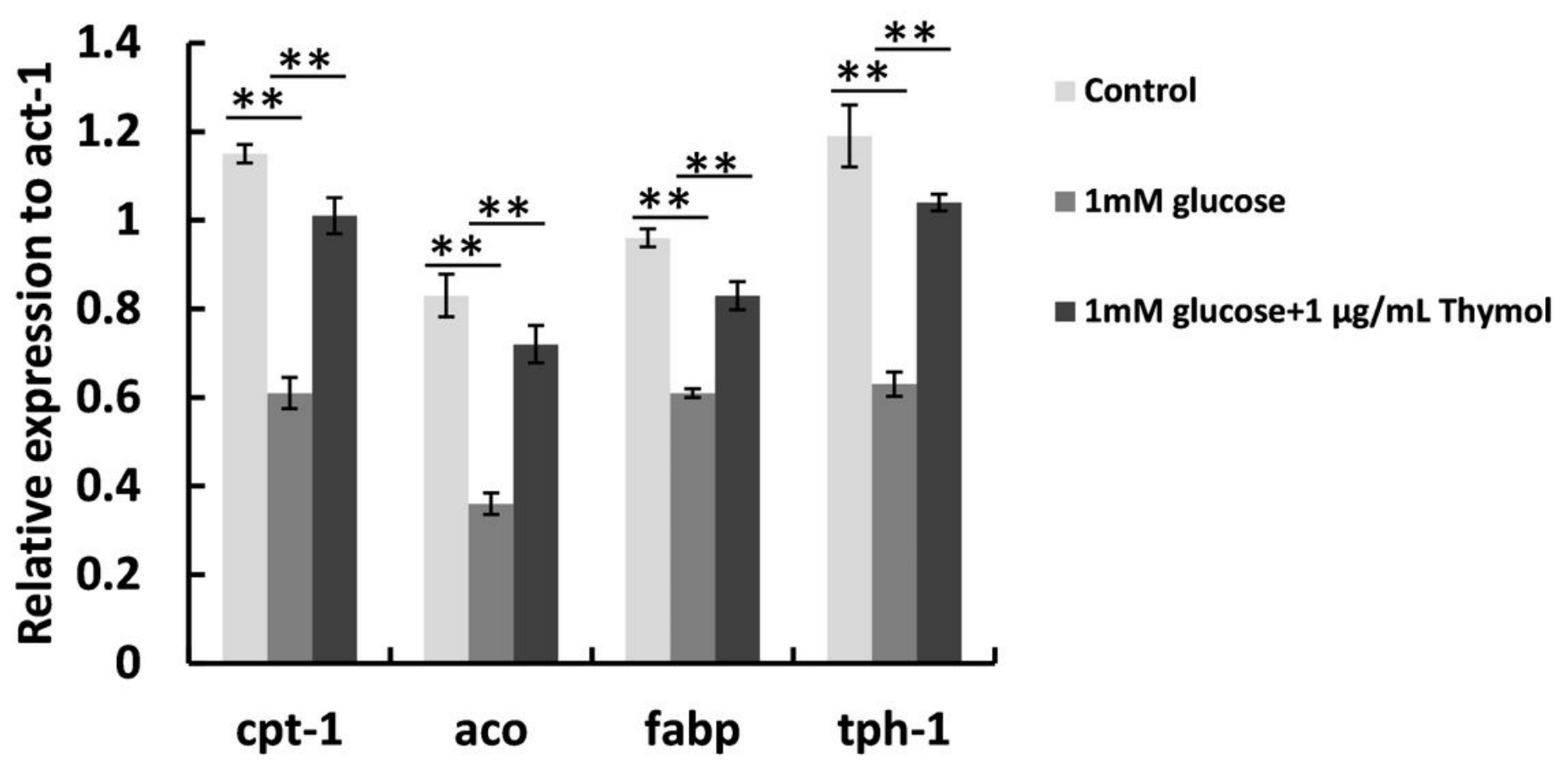

Figure 4

Transcriptional level of serotonin synthesis gene tph- 1 and $c p t-1$, aco, fabp in the process of $\beta$-oxidation for the group of N2, glucose treatment, and thymol treatment, ${ }^{*}<<0.05,{ }^{*} \mathrm{P}<0.01$. 


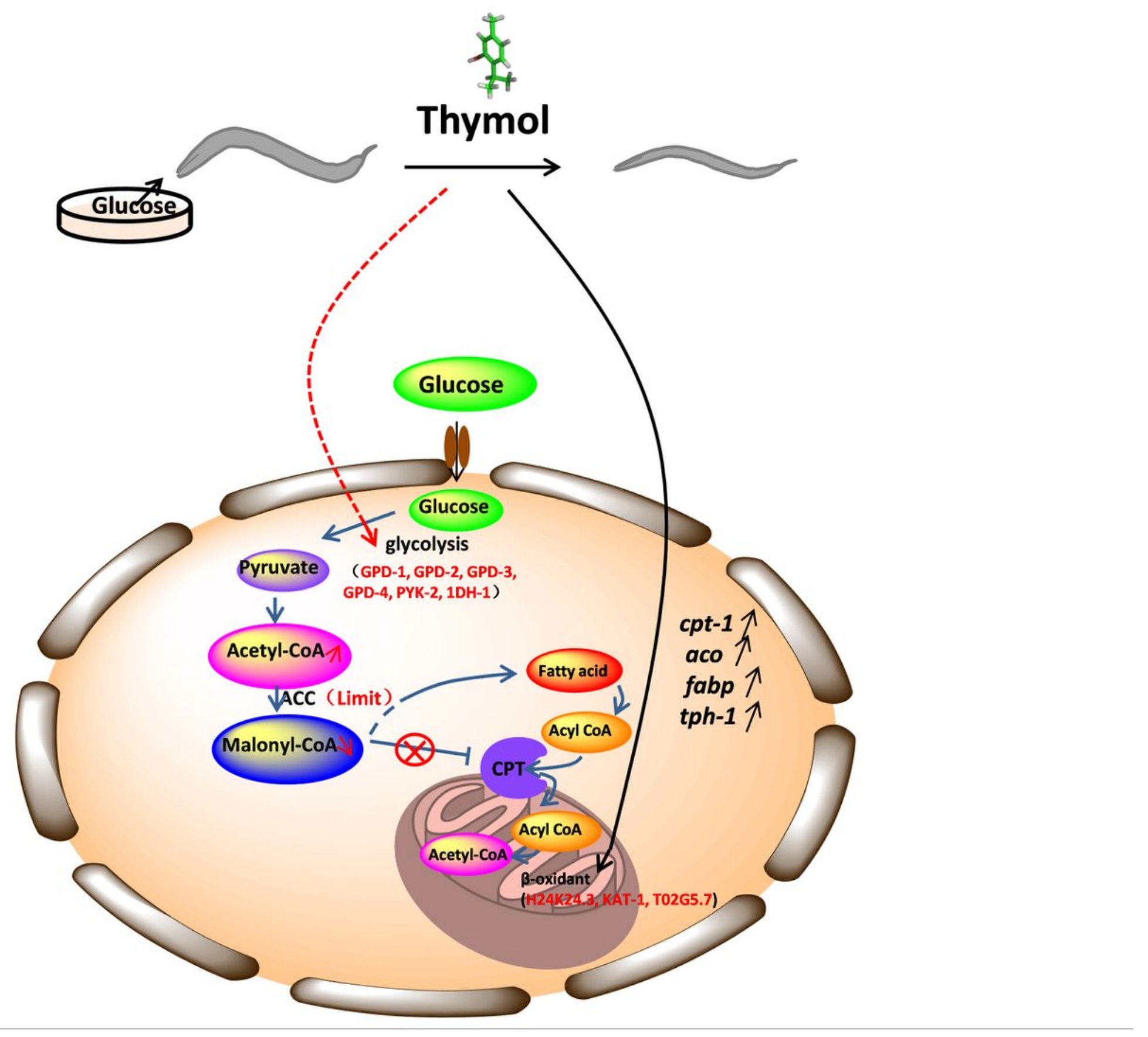

Figure 5

Diagram of thymol alleviating the obesity induced by glucose. 